\title{
Reporte de un caso de Cistinuria: Uso de herramientas de diagnóstico a nivel nacional
}

\author{
*Rosa Guillén ${ }^{1,3,4}$, José Blasco², Patricia Funes ${ }^{3}$, Dominich Granado ${ }^{3}$ \\ ${ }^{1}$ Hospital Central, Instituto de Previsión Social. Laboratorio de Urgencias. Paraguay \\ ${ }^{2}$ Hospital Central, Instituto de Previsión Social. Servicio de Urología. Paraguay \\ ${ }^{3}$ Universidad Nacional de Asunción, Instituto de Investigaciones en Ciencias de la Salud. San Lorenzo, Paraguay \\ ${ }^{4}$ Universidad Nacional de Asunción, Facultad de Ciencias Médicas, Cátedra de Bioquímica. San Lorenzo, Paraguay
}

Cómo referenciar este artículo/ How to reference this article:
Guillén R, Blasco J, Funes P, Granado D. Reporte de un caso de Cistinuria: Uso de herramientas de diagnóstico a nivel nacional. Mem. Inst. Investig. Cienc. Salud. $2018 ; 16(2): 94-99$

\section{R E S U M E N}

La cistinuria es una enfermedad genética que se engloba dentro de alteraciones congénitas del transporte de aminoácidos con formación de cálculos en las vías urinarias, si bien es poco frecuente se caracteriza por su elevada recurrencia. En este trabajo presentamos el caso de una paciente de 34 años, con antecedentes de haber perdido un riñón por episodios anteriores de litiasis y con múltiples recidivas que es diagnosticada mediante la detección de cistina por espectroscopía infrarroja como componente único de 96 fragmentos de cálculos removidos mediante nefrolitotomía percutánea. La paciente fue evaluada laboratorialmente mediante el perfil metabólico y la cristaluria. Las indicaciones de tratamiento específicas incluyeron la administración de agentes alcalinizantes, régimen nutricional, y entrenamiento para control de $\mathrm{pH}$ urinario. Es importante señalar la agresividad de la litiasis de cistina con las consecuencias que puede tener la calidad de vida del paciente, y por tanto la importancia de contar con capacidades instaladas a nivel país para el diagnóstico y seguimiento de litiasis genéticas como la causada por la cistinuria.

Palabras clave: cistinuria, Litiasis urinaria, enfermedad renal genética.

\section{Report of a case of cystinuria: Use of diagnostic tools at national level}

\section{A B S T R A C T}

Cystinuria is a genetic disease that is included among congenital defects of renal amino acids transport that causes urinary stone formation. Although it is rare, it is characterized by its high recurrence. We present the case of a 34-year-old patient that lost one of her kidney because of recurrent episodes of lithiasis, and that was diagnosed by the detection of cystine with infrared spectroscopy as the sole component of 96 stone fragments removed by percutaneous nephrolithotomy. The patient was evaluated by metabolic profile and crystalluria. The specific treatment indications included the administration of alkalinizing agents, nutritional regimen, and training for personal measurement of urinary $\mathrm{pH}$. This case highlights the aggressiveness of cystine stones with the consequences that may have on the quality of the patient life, and therefore the importance of having installed proper diagnostic capacities at national level to detect and monitor treatment efficacy in genetic lithiasis such as cystinuria.

Keywords: cystinuria, urolithiasis, genetic kidney disease.

\section{INTRODUCCIÓN}

La cistinuria es una enfermedad genética que se engloba dentro de alteraciones congénitas del transporte de aminoácidos, como consecuencia de esto se observa un aumento de la excreción urinaria de cistina, ornitina, lisina y arginina. Si bien se incrementan los niveles urinarios de varios aminoácidos, es el aumento de los niveles de Fecha de recepción: febrero 2018. Fecha de aceptación: marzo 2018

*Autor correspondiente: Dra. Rosa Guillén. Universidad Nacional de Asunción, Instituto de Investigaciones en Ciencias de la Salud. San Lorenzo, Paraguay. Dr Cecilio Baez c/ Dr Villamayor, Campus Universitario de la UNA, San Lorenzo; 0994318520. 
cistina el que conduce a la formación de cálculos urinarios debido a su baja solubilidad. La cistinuria es una enfermedad hereditaria, como consecuencia de mutaciones en los genes SLC3A1 y SLC7A9 ubicados respectivamente en los cromosomas 2 y $19^{(1,2)}$.

El debut de la sintomatología litiásica puede observarse en la primera década de la vida (25-30\%), la adolescencia (25-30\%) o en la adultez y afecta por igual a ambos sexos. Las litiasis de cistina son relativamente poco frecuentes, correspondiendo entre el 1 al $2 \%$ de todas las formas de urolitiasis en adultos, mientras que en niños su prevalencia puede alcanzar entre el $6 \%$ al $8 \%{ }^{(3-5)}$.

La repercusión renal que presentan los pacientes con cistinuria se centra en el riesgo de presentar hipofunción e insuficiencia renal. Esto sumado a la elevada tasa de recurrencia hacen que sea imprescindible un estricto seguimiento de los pacientes con litiasis de cistina (6). La presencia o ausencia de cristaluria y el volumen de los cristales de cistina correlacionan con la actividad de la litiasis y predicen el nivel de recurrencia ${ }^{(7)}$.

Una característica de los cálculos de cistina es la gran dureza, por lo que la litotricia extracorpórea por ondas de choque (LEOC) no siempre brinda buenos resultados, necesitando con frecuencia múltiples sesiones y tratamientos complementarios como la nefrolitotomía percutánea (NLPC) y disolución mediante irrigación. El diagnóstico de la cistinuria se basa en el hallazgo de cristales en orina o bien por la confirmación de la presencia de cistina como componente de cálculos urinarios ${ }^{(8)}$.

En el presente trabajo presentamos un caso clínico de litiasis por cistinuria diagnosticado mediante el empleo de herramientas laboratoriales disponibles en el país.

\section{PRESENTACIÓN DEL CASO}

Una paciente de sexo femenino, de 34 años de edad, $67 \mathrm{~kg}$ de peso y $1.64 \mathrm{~m}$ de estatura procedente de Luque consulta en el año 2009 en el Instituto de Previsión Social por cólicos renoureterales, se realiza estudio ecográfico y se constata litiasis coraliforme bilateral, hidronefrosis derecha.

En abril del 2010, acude al servicio de urgencias con dolor de fosa lumbar derecha, refiere ser portadora de catéter doble jota desde 7 meses atrás con cambio del lado derecho 18 días atrás. Queda internada para estudio y tratamiento de pielonefritis aguda. Se programa nefrectomía simple derecha por hallazgo de litiasis piélica derecha de $4,5 \mathrm{~cm}$ y pielonefrosis lado derecho. Se remite al Servicio de Anatomía patológica pieza quirúrgica correspondiente a riñón derecho de $13.5 \times 8 \times 6 \mathrm{~cm}$ resultado el diagnóstico pionefrosis, pielonefritis aguda y crónica severa y uretritis aguda y crónica.

Posteriormente en el 2011 se realiza litotricia extracorpórea como tratamiento para litiasis recidivante ubicada en riñón izquierdo, sin éxito terapéutico. En abril del 2016 se realiza NLPC izquierda por cálculo coraliforme, extrayendo aproximadamente $80 \%$ de la masa litiásica. Como resultado de este último procedimiento se remitieron al laboratorio de Urgencias del Hospital Central de IPS, 96 fragmentos de cálculo que fueron sometidos al análisis morfológico arrojando como resultado una morfología caracterizada por cristales de color marrón claro, aspecto traslúcido y agrupación más o menos laxa en formato radial, típica de la clase Va de la clasificación morfoconsitucional publicada por Daudon et al. ${ }^{(9)}$, compuesta por cistina. Los resultados morfológicos fueron confirmados, en la cátedra de Bioquímica de la FCM, por espectrofotometría infrarroja con transformada de Fourier con el equipo Prestige 21 (Shimadzu, Japón), empleando la metodología de la pastilla de $\mathrm{KBr}$ con pureza grado FTIR (Merck, USA). El espectro FTIR obtenido fue comparado con bases de datos y confirmaron la composición del cálculo de cistina (Figura 1). 
A

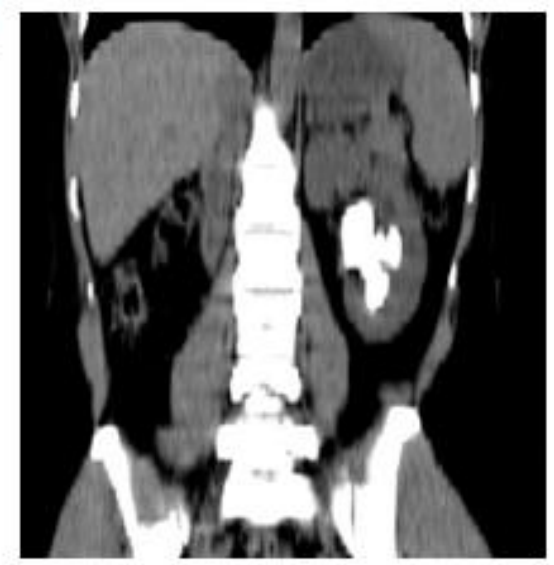

B

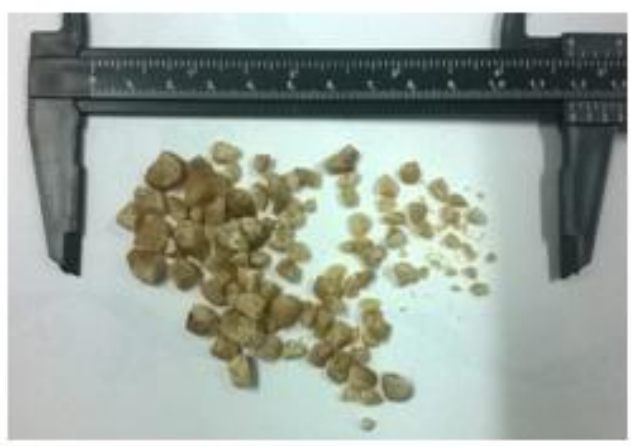

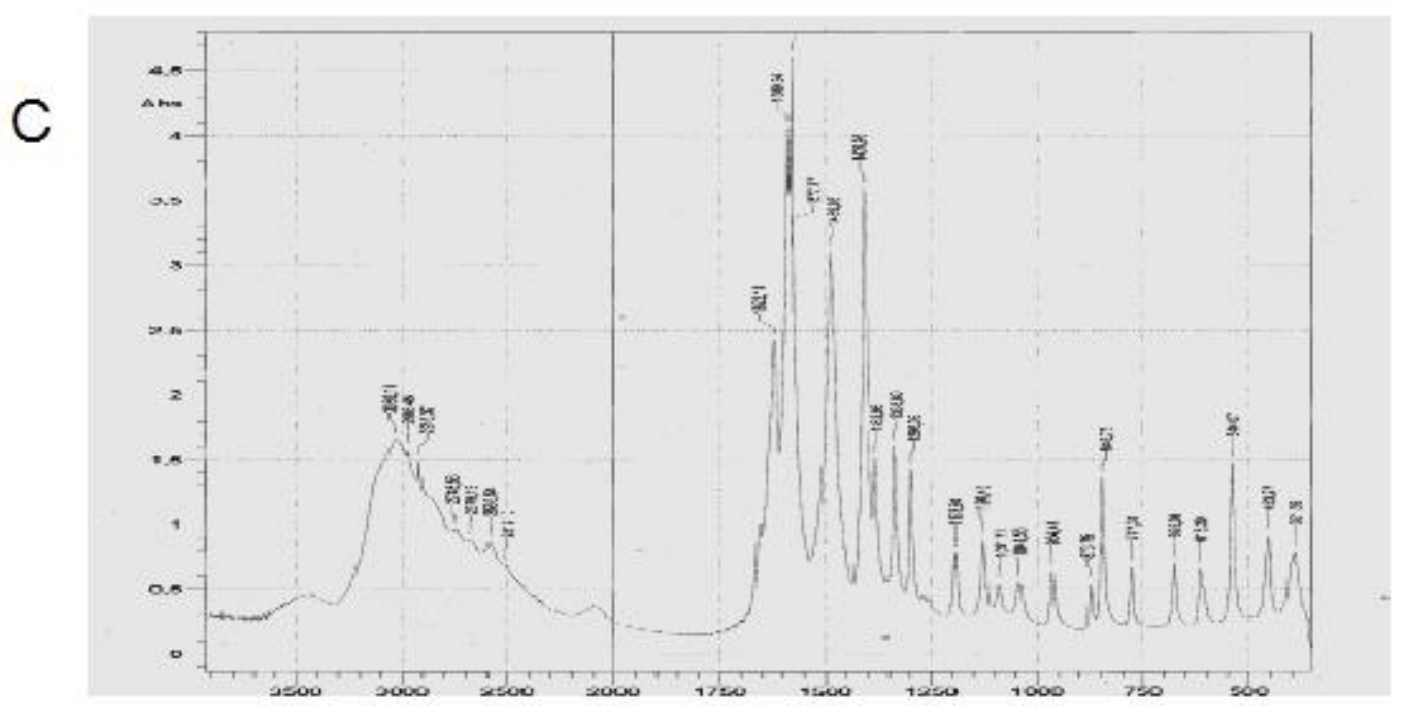

Figura 1. Fragmentos de cálculo renal obtenido por NLPC. A) Tomografía computarizada abdominal, muestra imagen de cálculo coraliforme previa a la NLPC realizada en el 2016 B) 96 fragmentos con

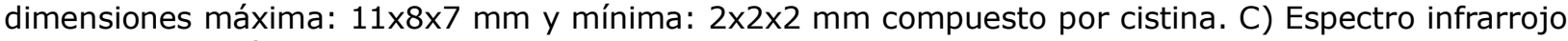
obtenido por análisis FTIR de los fragmentos.

A fin de realizar una evaluación metabólica de la paciente, así como el análisis de riesgo de recidiva basal, se sometió a la paciente a estudio metabólico de litiasis renal y estudios de cristaluria, ambos en el Instituto de Investigaciones en Ciencias de la Salud, en junio del 2016. El perfil metabólico de la paciente mostró como valores fuera de rango la calcemia y la magnesemia, mientras que los demás analitos tanto en sangre como en orina de 24 horas se encontraron dentro de los valores normales (Tabla 1 ).

Tabla 1. Valores séricos y urinarios de analitos litogénicos

\begin{tabular}{lclc}
\hline En suero & Resultados & En orina 24 Hs & Resultados \\
\hline Calcio & $12 \mathrm{mg} / \mathrm{dL}$ & Calcio & $88 \mathrm{mg} / 24 \mathrm{hs}$ \\
Magnesio & $1.1 \mathrm{mg} / \mathrm{dL}$ & Magnesio & $72 \mathrm{mg} / 24 \mathrm{hs}$ \\
Fosforo & $2.6 \mathrm{mg} / \mathrm{dL}$ & Fosforo & $541 \mathrm{mg} / 24 \mathrm{hs}$ \\
Ácido úrico & $4.6 \mathrm{mg} / \mathrm{dL}$ & Ácido úrico & $324 \mathrm{mg} / 24 \mathrm{hs}$ \\
Urea & $34 \mathrm{mg} / \mathrm{dL}$ & Urea & $21 \mathrm{~g} / 24 \mathrm{hs}$ \\
Creatinina & $1.04 \mathrm{mg} / \mathrm{dL}$ & Creatinina & $18 \mathrm{mg} / \mathrm{kg} / 24 \mathrm{hs}$ \\
& - & Citrato & $202 \mathrm{mg} / 24 \mathrm{hs}$ \\
& - & Sodio & $144 \mathrm{mEq} / 24 \mathrm{hs}$ \\
& - & Potasio & $55 \mathrm{mEq} / 24 \mathrm{hs}$ \\
\hline
\end{tabular}

Se realizó el estudio de cristaluria en dos días seguidos empleando muestras de orina excretadas en ayunas. En este estudio es importante resaltar que se realiza el análisis de la 
presencia de cristales, su identificación, cuantificación y medición precisa en dos etapas dentro de las 2 primeras horas de emitida la muestra y luego de las 48 horas conservándola a $4^{\circ} \mathrm{C}$. Los resultados de la primera lectura evidencian el riesgo de cristalización en el momento exacto del estudio y los resultados de la segunda muestra ponen de manifiesto riesgo que podrían presentarse en condiciones que favorezcan la cristalización o bien la presencia de especies cristalinas poco frecuentes en situación límite de sobresaturación. En el caso de la paciente en cuestión la primera lectura no arrojó la presencia de cristales, sin embargo, éstos si fueron evidentes en la segunda lectura (Figura 2).

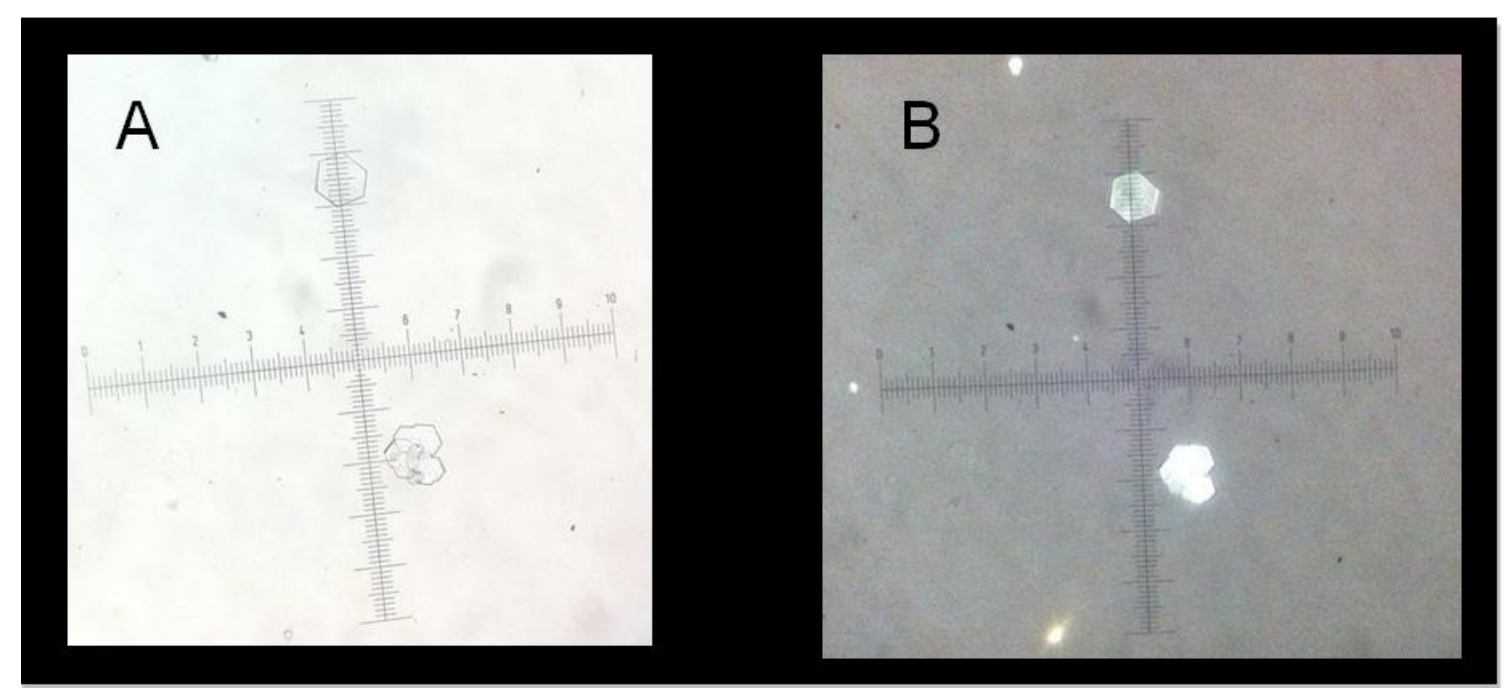

Figura 2. Cristales de cistina en orina emitida en ayunas. a) Microscopía con luz blanca b) Microscopía con luz polarizada.

La paciente recibió la indicación de un régimen nutricional con restricción de la ingesta de proteínas y el aumento de la ingesta de líquido para obtener la dilución de la orina por incremento de la diuresis. También se le recomendó realizar el control del pH urinario diario mediante el uso de las tiras reactivas en su domicilio. Para el uso de las mismas la paciente recibió un entrenamiento personalizado en el laboratorio de urgencias de IPS. Este factor es clave para evitar la formación de cristales de cistina ya que los mismos alcanzan su mayor solubilidad a pH neutro y por tanto es de extrema utilidad para medir la eficacia real de los agentes alcalinizantes empleados en el tratamiento de este tipo de litiasis.

\section{DISCUSIÓN}

La litiasis por cistina es un tipo de litiasis génica, constituye el 1 a $5 \%$ de las litiasis y se caracteriza por ser altamente recurrente pudiendo formar varios cálculos en un año de tamaño y morfología variable ${ }^{(10,11)}$. En nuestra experiencia el caso que presentamos es el tercero registrado por nuestro grupo de investigación en más de 1200 pacientes litiásicos que han remitido cálculos para el análisis morfológico, de forma coincidente con lo reportado a nivel mundial ${ }^{(12)}$.

Es importante resaltar la evolución extremadamente agresiva de la patología litiásica en la paciente. Esta debuta con la pérdida de uno de los riñones como consecuencia de la litiasis y posteriormente sufre de episodios recidivantes que han requerido repetidos procedimientos urológicos con escaso éxito terapéutico en el caso de la litotricia extracorpórea y con mejores resultados en casos de procedimientos más invasivos con la NLPC. La literatura refiere que los cálculos de cistina debido a su estructura cristalina compacta es de cierta manera muy resistente a la fragmentación por LEOC, de hecho este tipo de tratamiento se recomienda sólo en casos de pequeños cálculos no mayores a 15 $\mathrm{mm}$. Mientras que la NLPC se recomienda para cálculos de cistina voluminosos situados en los riñones o múltiples localizados en el cáliz inferior ${ }^{(8,13)}$.

La rapidez de formación de los cálculos de cistina junto a la necesidad de tratamientos urológicos invasivos pone en evidencia la importancia primordial del tratamiento en estos pacientes. El tratamiento de este tipo de patología se divide en medidas nutricionales con la meta de disminuir el aporte de metionina en los alimentos mediante una dieta con 
restricción en la ingesta de proteínas. La disminución del consumo de se acompaña de una disminución de la cistinuria ${ }^{(14)}$. Otra medida fundamental es la regulación del pH urinario, alcalinizando hasta un valor de $\mathrm{pH} 7,5$ para alcanzar la mayor solubilidad de la cistina, así como un aumento marcado de la diuresis de alrededor de $4 \mathrm{~L} /$ día $^{(7,8,15)}$.

Para poder iniciar las medidas terapéuticas específicas con la mayor rapidez posible es esencial hacer uso de las herramientas de diagnóstico como el análisis del cálculo y la determinación de la cristaluria. Ambos tipos de determinaciones se encuentran disponibles a nivel nacional y han sido empleadas de forma exitosa en el diagnóstico y seguimiento del tratamiento de un caso de cistinuria publicado anteriormente y cuya evolución ha sido muy buena teniendo a una paciente libre de recidivas por más de dos años ${ }^{(16)}$.

La eficacia de la dilución urinaria así como del tratamiento alcalinizante debe ser muy regular con la medición periódica de la densidad del pH urinario teniendo como metas una densidad menor a 1,010 y un pH urinario de 7,5. La desaparición de cristales de cistina en las orinas de mañana es uno de los mejores criterios para medir la eficacia del tratamiento (17). En este aspecto al analizar los resultados de la cristaluria se puede notar que a pesar de que en la lectura inicial no se observaron cristales de cistina, los mismos si aparecieron después del almacenamiento y además, resulta importante destacar la importancia del control seriado de cristalurias. Varios autores señalan a la cristaluria como un buen índice del riesgo de recidiva incluyendo el cálculo de índices como la determinación del volumen cristalino global es la mejor manera de conocer la actividad litogénica. Si el volumen cristalino global para la cistina es superior a $3000 \mu \mathrm{m}^{3 /} \mathrm{mm}^{3}$ el riesgo de litiasis es muy alto ${ }^{(17,18)}$.

Este caso pone de manifiesto la agresividad de la litiasis de cistina y las graves consecuencias que puede tener para el paciente si no es diagnosticado a tiempo. Así mismo podemos reportar que a nivel nacional se cuenta con el acceso a las determinaciones especializadas necesarias para confirmar el diagnóstico como el análisis del cálculo y hacer el seguimiento de la eficacia del tratamiento de una forma sencilla, práctica y a bajo costo mediante análisis de cristaluria. Estas técnicas laboratoriales especializadas brindan información esencial para el diagnóstico etiológico en específicos en cada caso y la toma de decisiones en pacientes con litiasis renal, además de constituir una herramienta para el seguimiento eficaz de los pacientes afectados con la patología.

\section{CONFLICTO DE INTERES}

Los autores declaran no presentar conflictos de intereses en la realización del presente trabajo.

\section{REFERENCIAS BIBLIOGRÁFICAS}

1. Orts Costa JA, Zúñiga Cabrera A, Martínez de la Cára y Salmerón ]. [Cystinuria update: clinical, biochemical and genetic aspects]. An Med Interna Madr Spain 1984. 2003 Jun;20(6):317-26.

2. Koulivand L, Mohammadi M, Ezatpour B, Kheirollahi M. Cystinuria in a patient with a novel mutation in SLC7A9 gene. Iran J Kidney Dis. 2015 Jan;9(1):63-6.

3. Daudon M. [Epidemiology of nephrolithiasis in France]. Ann Urol. 2005 Dec;39(6):20931.

4. Tiselius H-G. Epidemiology and medical management of stone disease. BJU Int. 2003 May;91(8):758-67.

5. Sharma AP, Filler G. Epidemiology of pediatric urolithiasis. Indian J Urol IJU J Urol Soc India. 2010 Oct;26(4):516-22.

6. Döven SS, Delibaş A, Taşkınlar $H, \mathrm{Naycı} \mathrm{A.}$ The impact of surgical intervention on renal function in cystinuria. J Bras Nefrol Orgao Of Soc Bras E Lat-Am Nefrol. 2018 Jun 21;

7. Jungers $P$, Joly D, Gagnadoux MF, Daudon M. [Cystine lithiasis: physiopathology and medical treatment]. Prog En Urol J Assoc
Fr Urol Société Fr Urol. 2001 Feb;11(1):122-6.

8. Jungers, Paul, Daudon, Michel, Cohort, Pierre. Lithiase rénale, Diagnostic et traitement. Paris: Flamarion; 1999. 226 p.

9. Daudon $M$, Bader CA, Jungers $P$. Urinary calculi: review of classification methods and correlations with etiology. Scanning Microsc. 1993 Sep;7(3):1081-104; discussion 1104-6.

10. Eggermann $T$, Venghaus A, Zerres $K$. Cystinuria: an inborn cause of urolithiasis. Orphanet J Rare Dis. 2012;7:19.

11. Martell HJ, Wong KA, Martin JF, Kassam Z, Thomas K, Wass MN. Associating mutations causing cystinuria with disease severity with the aim of providing precision medicine. BMC Genomics. 2017 $11 ; 18$ (Suppl 5):550.

12. Sumorok N, Goldfarb DS. Update on cystinuria. Curr Opin Nephrol Hypertens. 2013 Jul;22(4):427-31.

13. Rousaud F, Gracia S, Palacín M, Nunes V, Millán $F$, Oliver $A$, et al. [Cystinuria and cystine kidney lithiasis. Diagnosis and 
therapeutic approach]. Arch Esp Urol. 2001 Nov; 54(9):989-96.

14. Nagaraja P, Premminger G, Kavangh J. Urinary tract stone disease. Londres: Springer; 2011.720 p.

15. Bouzidi $H$, Daudon M. [Cystinuria: from diagnosis to follow-up]. Ann Biol Clin (Paris). 2007 Oct;65(5):473-81.

16. Guillén, R, Funes, P, Ruiz, I, Duarte, C, Mereles, B. Litiasis por cistina: Reporte de un caso. Mem Inst Investig Cienc Salud. 2014;12(1):51-6.
17. Daudon M, Cohen-Solal F, Barbey F, Gagnadoux M-F, Knebelmann B, Jungers $P$. Cystine crystal volume determination: a useful tool in the management of cystinuric patients. Urol Res. 2003 Jul;31(3):207-11.

18. Wong KA, Pardy C, Pillay S, Athanasiou T, Rottenberg G, Bultitude M, et al. Can the Presence of Crystalluria Predict Stone Formation in Patients with Cystinuria? J Endourol Endourol Soc. 2016 May;30(5):609-14. 